\title{
Mini-Review
}

\section{Acute Complicated Brucellosis Mimicking Crimean-Congo Hemorrhagic Fever (CCHF) and Vice Versa}

\author{
Ali Akbar Heydari ${ }^{*}$ \\ ${ }^{1}$ Department of Infectious Diseases and Research Center for Infection Control and Hand Hygiene, Imam Reza \\ Hospital, Mashhad University of Medical Sciences, Mashhad, Iran
}

Received Apr. 17, 2019; Accepted Jul. 20, 2019

\begin{abstract}
Brucellosis and Crimean Congo Hemorrhagic Fever (CCHF) are both common zoonoses that may co-occur in similar epidemiological conditions, e.g., among young livestock breeders, veterinarians, and farmers in rural areas. Transmission of Brucella bacteria is through ingestion of contaminated dairy products, while CCHF virus infection occurs via infective tick bite or exposure of damaged skin and mucosa to the tissues and blood of viremic animals. Brucellosis occurs almost in all seasons, while CCHF is of lower incidence in the cold seasons due to decreased activity of tick vectors. CCHF mimics brucellosis and vice versa, mainly when the latter manifests severe thrombocytopenia and hemorrhage. Occasionally, the two illnesses present similar clinical features and laboratory results, e.g., fever, muscle aches, increased liver enzymes, and thrombocytopenia. This article discusses the similar clinical, epidemiological and laboratory aspects of the two diseases and warns physicians to avoid the inappropriate use of drugs such as ribavirin, which is dangerous in patients with kidney failure and pregnancy. $J$ Med Microbiol Infec Dis, 2019, 7 (1-2): 1-5.
\end{abstract}

Keywords: Thrombocytopenia, Brucellosis, Crimean-Congo Hemorrhagic Fever.

\section{INTRODUCTION}

Crimean-Congo hemorrhagic fever (CCHF), endemic to Asia, Africa, and southern Europe, is a tick-borne disease caused by the CCHF virus (Orthonairovirus in Nairoviridae family). The main route of transmission is tick bite, and close contact with virus-infected blood/tissues of humans or animals may also transmit the infection. $\mathrm{CCHF}$ is considered a significant public health problem with case fatality rate (CFR) of 5.4-80\% [1].

$\mathrm{CCHF}$ remains a seasonal disease and is rare in winter. In the case of similar epidemiologic and clinical presentation, brucellosis should be taken into consideration in endemic regions for the differential diagnosis of thrombocytopenia associated bleeding (with or without hemophagocytosis), because this picture is not rare in the latter illness [2].

After a few days (usually 2 to 14 days) incubation period, CCHF presents with nonspecific symptoms of fever, headache, body pain, and vomiting. During the hemorrhagic phase, petechia, ecchymosis, and bleeding from the various mucosal surface of the body, including respiratory tracts, genitourinary, and gastrointestinal tract can occur [3]. Severe CCHF can result in disseminated intravascular coagulation (DIC), shock, and death.

Brucellosis, a prevalent zoonotic infection, is endemic to different regions of Mediterranean countries, the Balkans, the Persian Gulf, the Middle East, and Central and South America with a predominance of the agricultural and rural population [4]. This infection, caused by Brucella spp. a Gram-negative bacteria mimics different non-infectious and infectious diseases, and its resemblance to other medical conditions can lead to increased mortality and morbidity as well as misdiagnosis and under-reporting of the disease. Human infection is via the secretions and fluids of infected animals. The bacteria enter the human body through direct contact with infected materials like afterbirth or the consumption of raw milk and dairy products and via inhalation of the bacteria. Human to humans transmission is infrequent. The brucellosis transmission can also occur through the blood transfusion, bone marrow transplantation, and sexual contact. Veterinarians, shepherd, and farmers, and laboratory staff are high at-risk groups [1].

In humans, Brucella infection has a variety of clinical manifestations, mainly involving the reticuloendothelial system [3]. After a 14-21 days incubation period, clinical manifestation like fever, fatigue, anorexia, and musculoskeletal pain may occur. The most common complaints associated with brucellosis are arthralgia, low back pain, fever, chills, malodorous sweating, anorexia, and

${ }^{*}$ Correspondence: Ali Akbar Heydari

Department of Infectious Diseases and Research Center for Infection Control and Hand Hygiene, Imam Reza Hospital, Mashhad University of Medical Sciences, Mashhad, Iran, 9137913316.

Email: heydariaa@mums.ac.ir

Tel/Fax: +98 (51) 38547243 
myalgia. Despite the treatable and nonfatal nature of brucellosis, it may involve nearly all body system, and the disease may present with complications such as thrombocytopenia. This complication can be severe with purpura and bleeding [2]. According to several studies [2-4], brucellosis may present with severe hemorrhagic fever similar to $\mathrm{CCHF}$, which requires isolation of the patients before definitive diagnosis. Therefore, brucellosis should be taken into consideration in endemic regions for the differential diagnosis of hematological conditions, such as pancytopenia, thrombocytopenia, hemolytic anemia, and disseminated intravascular coagulation [5-6]. Table 1 shows the clinical and laboratory characteristics of $\mathrm{CCHF}$ and acute Brucellosis.

Table 1. Transmission route and clinical and laboratory characteristics of acute Brucellosis and CCHF

\begin{tabular}{|c|c|c|c|}
\hline \multicolumn{3}{|c|}{ CCHF } & Brucellosis \\
\hline \multicolumn{4}{|c|}{ Etiology } \\
\hline \multicolumn{3}{|c|}{ Orthonairovirus in Nairoviridae family } & Brucella bacterium (B.melitensis, B.abortus, B.suis, B.canis) \\
\hline \multicolumn{4}{|c|}{ Epidemiology } \\
\hline Distribution & \multicolumn{2}{|c|}{$\begin{array}{l}\text { Widespread geographic distribution (esp Africa, Middle East, } \\
\text { Europe, and Asia }\end{array}$} & Global distribution worldwide (except the few developed countries) \\
\hline Age & \multicolumn{2}{|c|}{ Any age (But more common in working-age population) } & Any age (especially 20-30 y) \\
\hline Gender & \multicolumn{2}{|c|}{$\mathrm{M}>\mathrm{F}(58 / 42)$} & $\mathrm{M}=\mathrm{F}$ \\
\hline Job & \multicolumn{2}{|c|}{$\begin{array}{l}\text { Slaughterhouses employees, animal handler/examiner } \\
\text { veterinarians, healthcare workers, farmer, shepherds }\end{array}$} & $\begin{array}{l}\text { Farmers, veterinarians, slaughterhouses employees' shepherds } \\
\text { Lab.Workers }\end{array}$ \\
\hline Season & \multicolumn{2}{|c|}{ Summertime ( between April and September ) } & All seasons (Mostly spring and summer) \\
\hline Transmission & \multicolumn{2}{|c|}{$\begin{array}{l}\text { Tick bite. Direct contact with CCHF virus-infected } \\
\text { blood/tissues of infected humans or animal }\end{array}$} & $\begin{array}{l}\text { Non-pasteurized milk and dairy products consumption, inhalation, } \\
\text { eye mucosa cutaneous lesions. Blood transfusion, bone marrow } \\
\text { transplantation, and sexual contact. }\end{array}$ \\
\hline \multicolumn{4}{|c|}{ Clinical Characteristics } \\
\hline \multicolumn{2}{|c|}{ Incubation period (days) } & $3-14$ & $7-28$ \\
\hline \multicolumn{2}{|c|}{ Fever $(\%)$} & 93 & $75-100$ \\
\hline \multicolumn{2}{|c|}{ Headache (\%) } & 73 & 56 \\
\hline \multicolumn{2}{|c|}{ Myalgia/arthralgia's (\%) } & 72 & 26 \\
\hline \multicolumn{2}{|c|}{ Diarrhea $(\%)$} & 33 & $3-6$ \\
\hline \multicolumn{2}{|c|}{ Hepatomegaly (\%) } & 30 & 17 \\
\hline & $10-30$ & 16 \\
\hline \multicolumn{3}{|c|}{ Splenomegaly (\%) } & eristics \\
\hline \multicolumn{3}{|r|}{$\frac{\text { Laboratory Charac }}{24}$} & 9 \\
\hline \multicolumn{2}{|c|}{ Leukopenia $(\%)$} & 10 & 11 \\
\hline \multicolumn{2}{|c|}{ Thrombocytopenia $(\%)$} & 86 & $1-26$ \\
\hline \multicolumn{2}{|c|}{ Elevated Transaminase $(\%)$} & $55 / 2-72$ & 25 \\
\hline
\end{tabular}

\section{Epidemiology}

CCHF. CCHF is a fatal hemorrhagic fever with a mortality of approximately $30 \%$. The disease was first characterized in Crimea in 1944 and later in Congo in 1956. A tick-borne virus belonging to the Orthonairovirus genus is the etiological agent of CCHF. The disease occurs in Africa, Asia, Southeast Europe, and some Middle Eastern countries, including Iran and Turkey [3].

This disease typically affects people who either work in close contact with animals, e.g., farmers, shepherds, veterinarians, and abattoirs workers or those handling specimens from patients in healthcare centers. An infected tick bite can also transmit the virus to humans. The disease is developed only in infected humans as the incidental host [7].

Brucellosis. More than 500,000 people are infected annually. Brucellosis is a common infection, which occurs in the laboratory setting and is categorized as a subgroup of bioterrorism. It is also one of the most significant health problems in some parts of Asia, Africa, Latin America, and Mediterranean regions [8].
Brucellosis is especially prevalent in Middle East countries. In this region, increased risk of brucellosis may be due to wars in some countries, unchecked animal import from other countries, and inadequate prevention and control measures [9]. Farmers, shepherds, veterinarians, and workers of abattoirs and laboratories may be occupationally exposed to this infection. Inhalation, ingestion, and mucosal/percutaneous exposure can result in brucellosis acquisition. Moreover, accidental injection of B. abortus vaccine strains may result in brucellosis [10].

\section{Pathogenesis}

CCHF. The pathophysiology of CCHF is not yet wellknown. Some studies showed that the virus, after replication in local tissues and dendritic cells, moves to regional lymph nodes and disseminates into the spleen, liver, and lymph nodes. Endothelial damage and impairment of the immune system contribute to the symptoms and signs of CCHF [11]. Cytokines, including interleukin- 6 and 8 and TNF- $\alpha$, increase in the blood, and their presence intensifies the severity of the disease. In severe and lethal cases of CCHF, serum levels of $\mathrm{IgG}$ are 
low or zero. In acute and fatal cases, a high serum level of inflammatory markers, such as ferritin and procalcitonin, have also been reported [11]. Thrombocytopenia is the most common hematologic finding in $\mathrm{CCHF}$ and occurs in almost all patients [12].

Some studies indicated an association between this infection and nonfatal and fatal fulminant thrombotic thrombocytopenic purpura [13]. Platelet, endothelial cell, and immunological and inflammatory pathway impairment may result in hemorrhagic events [14]. Cytopenia observed in cases of $\mathrm{CCHF}$ may be due to apoptosis. Furthermore, hemophagocytosis in the bone marrow of CCHF patients can be indicative of apoptosis in the bone marrow. Apoptosis may be widespread in the liver, brain, and endothelial cells. Moreover, multiorgan failure can be due to hemophagocytic activity, triggered by widespread apoptosis [15].

CCHF-induced bleeding has been attributed to bone marrow suppression, liver dysfunction, and disseminated intravascular coagulation (DIC) [16]. The contribution of genetic factors on $\mathrm{CCHF}$ have been evaluated. In comparison with healthy people, CCHF patients showed a significantly higher HLA-A*02 frequency. Nonetheless, HLA-B*27 was significantly lower in individuals infected with $\mathrm{CCHF}$ than the control population. The genetic biomarkers suggested as prognostic factors for $\mathrm{CCHF}$ include cytokine gene polymorphisms, nuclear factor genetic polymorphisms, and Toll-like receptors [11].

Brucellosis with an emphasis on thrombocytopenia. Severe thrombocytopenia is uncommon despite the occurrence of mild thrombocytopenia in $1-26 \%$ of patients with brucellosis [17]. Brucella associated thrombocytopenia occurs due to the suppression of the bone marrow, hemophagocytosis, hypersplenism, immunological cell destruction, and DIC [4]. Moreover, thrombocytosis is known as an inflammation marker, although the low number of platelets may also be a sign of infection-induced immunosuppression and severe infection [18].

Microbial endotoxin may result in platelet adhesion, endothelial damage, and platelet removal from the blood [19]. Immunological destruction of platelets is induced by thrombocytopenia. This mechanism is confirmed by the fact that the majority of patients with brucellosis have a positive Coombs test, respond to corticosteroid treatment, and are test positive for antiplatelet antibodies [20]. Overall, production failure in the bone marrow damaged peripheral circulation, and trapped or enlarged spleen may result in low platelet count in brucellosis.

In response to infectious diseases (e.g., brucellosis), thrombocytopenia may occur due to hypersplenism, hemophagocytosis, bone marrow suppression, the formation of granuloma in the bone marrow, and DIC [2124]. In a previous study, granuloma formation and hypercellular bone marrow were reported in $41 \%$ and $75 \%$ of the samples, respectively, according to the bone marrow biopsy [23]. Moreover, splenomegaly was detected in $75 \%$ of cases with hypercellular bone marrow and thrombocytopenia.
Active histiocytes contribute to leukophagocytosis, erythrophagocytosis, and platelet phagocytosis in hemophagocytosis. Hemophagocytosis is reported in brucellosis, as well as various infectious and noninfectious diseases [24]. The bone marrow can be hypocellular or hypercellular despite the presence of thrombocytopenia with adequate megakaryocyte count [23, 25]. Thrombocytopenia cannot explain bone marrow suppression; therefore, other etiologies should be considered. Autoimmune phenomena due to Brucella bacteria are a significant cause of thrombocytopenia, leading to purpura, bleeding, and hemolytic anemia [26]. On the other hand, cryoglobulinemia and monoclonal hypergammaglobulinemia are rare complications of brucellosis [27].

\section{Clinical Manifestations}

CCHF. CCHF presents with a sudden fever, headache, and myalgia. Abdominal pain, vomiting, nausea, conjunctivitis, and bradycardia may also occur. In severe and progressive forms of the disease, various bleeding presentations, such as epistaxis, hematemesis, melena, hematuria, and hemoptysis, may also be reported. The major manifestations of CCHF include purpura, petechiae, ecchymosis, epistaxis, and bleeding from the genitourinary, respiratory, and gastrointestinal tracts.

In a previous study, hemorrhage, leukocytosis, melena, somnolence, petechiae, gum bleeding, ecchymosis, hematuria, hematemesis, hemoptysis, and hematoma were prevalent in the fatal group compared to the nonfatal group [28]. In some cases, central nervous system dysfunction, delirium, convulsion, and coma can develop. Typical laboratory findings include thrombocytopenia, increased prothrombin time (PT), and elongated activated partial PT [29].

Brucellosis with an emphasis on hemorrhagic diathesis. Brucellosis may compromise any body organ or system. The most common symptoms are fever, splenomegaly, hepatomegaly, peripheral arthritis, lymphadenopathy, and scrotal swelling [30].

The incidence of splenomegaly is higher in thrombocytopenic patients in comparison with those with uncomplicated brucellosis. Severe thrombocytopenia, hemoptysis, bleeding, and hematemesis are rare in patients with Brucella-induced thrombocytopenia [31]. Microangiopathy and thrombocytopenic purpura may also occur in brucellosis [32, 33]. Microangiopathic hemolytic anemia (MAHA) is rare in cases of Brucella endocarditis; however, clinical presentations of the mild form of this disease can lead to thrombosis, chronic bleeding, and even death [34]; DIC is also a rare outcome [35].

When evaluating a patient with potential thrombocytopenia, peripheral blood smears should be prepared for examining the blood cell morphology and excluding pseudo thrombocytopenia, particularly in patients with an unresolved low platelet count. The latter condition is related to certain anticoagulants and platelet agglutination [36]. 
Bone marrow culture and smear have significant contributions to the detection of infectious causes of thrombocytopenia. Nonetheless, less invasive procedures can determine the etiology of thrombocytopenia. In many cases, thrombocytopenia disappears after short-term antibiotic treatments. Therefore, no bone marrow examination is needed to confirm the diagnosis in suspected cases of infection-induced thrombocytopenia. In the differential diagnosis of brucellosis, hemorrhagic fever in endemic regions, idiopathic thrombocytopenic purpura (ITP), and hematologic malignancies should be taken into account, even in afebrile cases upon admission [37].

Brucellosis and CCHF are common diseases in rural areas, affecting shepherds and farmers. They can cause thrombocytopenia, fever, and bleeding (sometimes asymptomatic). The leading cause of the difference between these diseases is the lower frequency of CCHF in winter that is due to decreased activity of the tick vector [38], while brucellosis affects patients throughout the year. Although acute CCHF commonly presents with severe thrombocytopenia and hemorrhagic manifestations such as melena [39], brucellosis rarely causes such complications. Serological tests for individuals who were in contact with the animals or patients blood, history of tick exposure, and polymerase chain reaction assay can assist in confirming the CCHF diagnosis.

To summarize, there is an overlap between brucellosis and CCHF in terms of thrombocytopenia and hemorrhagic presentations and laboratory parameters. As most brucellosis patients with severe thrombocytopenia are erroneously treated with ribavirin due to the wrong diagnosis of CCHF, it is imperative that these patients undergo rapid tests for brucellosis before the initiation of ribavirin antiviral drug, especially if there is a risk of significant injury, i.e., renal failure. Severe thrombocytopenia might occur in cases of brucellosis and mimic CCHF. In the differential diagnosis of complicated brucellosis, hemorrhagic fever, ITP, and hematological malignancies should be taken into account in endemic regions.

\section{CONFLICT OF INTEREST}

The authors declare that there are no conflicts of interest associated with this manuscript.

\section{REFERENCES}

1. Duygu F, Sari T, Kaya T, Nermin Bulut. Brucellosis in Patients with Crimean-Congo Hemorrhagic Fever. J Arthropod Borne Dis. 2017; 11 (4): 463-468.

2. Heydari AA, Ahmadi F, Sarvghad MR, Safari H, Bajouri A, Saeidpour M. Hemophagocytosis and pulmonary involvement in brucellosis. Int J Infect Dis. 2007; 11: 89-90.

3. Metin O, Teke TA, Gayretli Aydin ZG, Kaman A, Oz FN, Bayhan GI, et al. A case of brucellosis mimicking CrimeanCongo hemorrhagic fever. J Infect Public Health. 2015; 8: 302304.

4. Al Mousa AI. Epistaxis as the initial manifestation of brucellosis. Int J Health Sci (Qassim). 2008; 2: 157-162.
5. Al-Eissa YA, Kambal AM, Al-Nasser MN, Al-Habib SA, AlFawaz IM, Al-Zamil FA.Childhood brucellosis: A study of 102 cases. Ped Infect Dis.1990; 9: 74-79.

6. Crosby E, Llosa L, Miro Quesada M, Carrillo C, Gotuzzo E. Hematologic changes in brucellosis. J Infect Dis. 1984; 150: 419-424.

7. Kilinc C, Gückan R, Capraz M, Varol K, Zengin E, Mengeloglu Z, Menekse E.J Vector Borne Dis. .Examination of the specific clinical symptoms and laboratory findings of Crimean-Congo hemorrhagic fever. 2016; 53(2): 162-7

8. Gwida M, Al Dahouk S, Melzer F, Rösler U, Neubauer H, Tomaso H. Brucellosis regionally emerging zoonotic disease? Croat Med J 2010; 51(4): 289-95

9. Gwida M, Al Dahouk S, Melzer F, Rösler U, Neubauer H, Tomaso H. Brucellosis - regionally emerging zoonotic disease? Croat Med J. 2010; 51(4): 289-95.

10. Ashford DA, di Pietra J, Lingappa J, Woods C, Noll H, Neville B, et al. Adverse events in humans associated with accidental exposure to the livestock brucellosis vaccine RB51. Vaccine. 2004 3; 22 (25-26): 3435-9.

11. Akinci E, Bodur H, Sunbul M, Leblebicioglu H. "Prognostic factors, pathophysiology and novel biomarkers in CrimeanCongo hemorrhagic fever" Antiviral research 2016; 132: 233243.

12. Sharififard M, Alavi SM, Salmanzadeh S, Safdari F, Kamali A. Epidemiological Survey of CrimeanCongo Hemorrhagic Fever (CCHF), Fatal Infectious Disease in Khuzestan Province, Southwest Iran, During 1999 - 2015. Jundishapur J Microbiol. 2016; 9 (5).

13. da Silva, Rodrigo Lopes. "Viral-associated thrombotic microangiopathies" Hematology/oncology and stem cell therapy 2011; 4 (2): 51-59.

14. Ergonul, Onder. "Crimean-Congo hemorrhagic fever virus: new outbreaks, new discoveries." Current opinion in virology 2012; 2 (2): 215-220.

15. Guler, Nil, Eroglu C, Yilmaz H, Karadag A, Alacam H, Sunbul M. et al. "Apoptosis-Related Gene Expression in an Adult Cohort with Crimean-Congo Hemorrhagic Fever" PloS one. 2016; 11 (6): e0157247.

16. S. K. Al-Tikriti, F. Al-Ani, F. J. Jurji, H. Tantawi, M. AlMoslih, N. Al-Janabi, , et.al. Congo/Crimean haemorrhagic fever in Iraq Bull World Health Organ. 1981; 59 (1): 85-90

17. Guzel Tunccan O, Dizbay M, Senol E, Aki Z, Ozdemir K. Isolated Severe Immune Thrombocytopenia due to Acute Brucellosis. Indian J Hematol Blood Transfus. 2014; 30: 27-29.

18. Al Mousa AI. Epistaxis as the initial manifestation of brucellosis. Int J Health Sci (Qassim). 2008; 2: 157-162.

19. de Stoppelaar SF, van 't Veer C, Claushuis TA, Albersen BJ, Roelofs JJ, van der Poll T. Thrombocytopenia impairs host defense in gram-negative pneumonia-derived sepsis in mice. Blood. 2014; 124: 3781-3790.

20. McCuskey RS, Urbaschek R, Urbaschek B. The microcirculation during endotoxemia. Cardiovasc Res. 1996; 32 : 752-763. 
21. Baldane S, Sivgin S, Alkan TS, Kurnaz F, Pala C, Keklik M, Karaman A, Kaynar L. An atypical presentation of brucellosis in a patient with isolated thrombocytopenia complicated with upper gastrointestinal tract bleeding. Case Rep Med. 2012; 2012: 473784.

22. García P, Yrivarren JL, Argumans C, Crosby E, Carrillo C, Gotuzzo E. Evaluation of the bone marrow in patients with brucellosis. Clinico-pathological correlation. Enferm Infecc Microbiol Clin. 1990; 8: 19-24.

23. Demir C, Karahocagil MK, Esen R, Atmaca M, Gönüllü H, Akdeniz H. Bone marrow biopsy findings in brucellosis patients with hematologic abnormalities. Chin Med J (Engl). 2012; 125 : 1871-1876.

24. George MR. Hemophagocytic lymphohistiocytosis: Review of etiologies and management.J Blood Med. 2014; 69-86.

25. Yildirmak Y, Palanduz A, Telhan L, Arapoglu M, Kayaalp $\mathrm{N}$. Bone marrow hypoplasia during Brucella infection. J Pediatr Hematol Oncol. 2003; 25: 63-4.

26. Pappas G, Kitsanou M, Christou L, TsianosE. Immune thrombocytopenia attributed to brucellosis and other mechanisms of Brucella-induced thrombocytopenia. Am J Hematol. 2004; 75: 139-141.

27. Yrivarren JL, Lopez LR. Cryoglobulinemia and cutaneous vasculitiesin human brucellosis.J Clin Immunol. 1987; 7: 471474.

28. Bastug A, Kayaaslan B, Kazancioglu S, Aslaner H, But A, Akinci E, et al. Crimean-Congo Hemorrhagic Fever: Prognostic Factors and the Association of Leukocyte Counts with Mortality. Jpn J Infect Dis. 2016; 69 (1): 51-5.

29. Kilinc C, Gückan R, Capraz M, Varol K, Zengin E, Mengeloglu Z,et.al.J Vector Borne Dis. Examination of the specific clinical symptoms and laboratory findings of CrimeanCongo hemorrhagic fever. 2016; 53 (2): 162-7.

30. H. Cem and Erdem H. Brucellosis (Brucella Species).in Mandell, Douglas, and Bennett's Principles and practice of
Infectious Diseases.Pub.Elsevier, 2015, 8 Ed. Chapter 228, Vol. 2, 2585-2589

31. Akıncı E, Bodur H, Erbay C, Cevik MA, Erbay A, Colpan A. A case of brucellosis presenting with severe thrombocytopenia. Turk J Hematol. 2003; 20: 43-45.

32. Young EJ, Tarry A, Genta RM, Ayden N, Gotuzzo E. Thrombocytopenic purpura associated with brucellosis: Report of 2 cases and literature review. Clin Infect Dis. 2000; 31: 904909.

33. Altuntas F, Eser B, Sari I, Yildiz O, Cetin M, UnalA. Severe thrombotic microangiopathy associated with brucellosis: Successful treatment with plasmapheresis. Clin Appl Thromb Hemost. 2005; 11: 105-108.

34. Madkour MM. Madkour's brucellosis. 2nd ed. New York: Springer-Verlag; 2001. 33-37

35. Turunc T, Demiroglu YZ, Kizilkilic E, Aliskan H, Boga C, Arslan H. A case of disseminated

intravascular coagulation caused by Brucella melitensis. J Thromb Thrombolysis. 2008; 26: 71-73.

36. Schrezenmeier $H$, Müller $H$, Gunsilius E, Heimpel $H$, Seifried E. Anticoagulant-inducedpseudothrombocytopenia and pseudoleucocytosis. Thromb Haemost. 1995; 73: 506-513.

37. evinc A, Buyukberber N, Camci C, Buyukberber S, Karsligil T. Thrombocytopenia in brucellosis: Case report and literature review. J Natl Med Assoc. 2005; 97: 290-293.

38. Köksal I, Yilmaz G, Iskender S, Arslan M, Yavuz I, Aksoy F. The first Crimean-Congohemorrhagic fever case in the winter season from Turkey. Vatansever ZIntervirology. 2011; 54: 144145 .

39. Mourya DT, Viswanathan R, Jadhav SK, Yadav PD, Basu A, Chadha MS . Retrospective analysis of clinical information in Crimean-Congo haemorrhagic fever patients: 2014-2015, India Indian J Med Res. 2017; 145 (5): 673-678. 